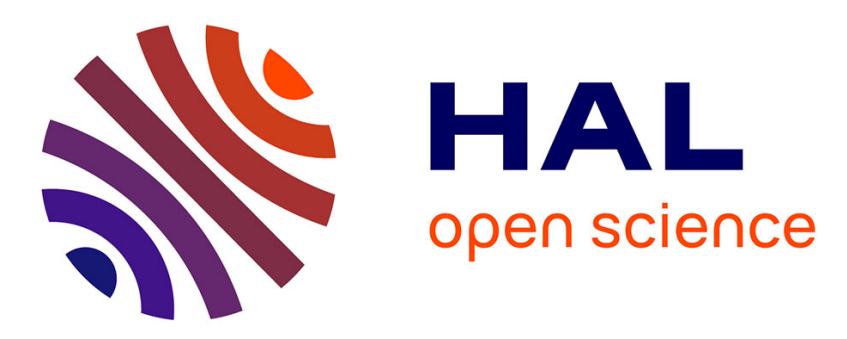

\title{
Blind Separation of rotating machine signals using Penalized Mutual Information criterion and Minimal Distortion Principle
}

Mohammed El Rhabi, Hassan Fenniri, Guillaume Gelle, Georges Delaunay

\section{- To cite this version:}

Mohammed El Rhabi, Hassan Fenniri, Guillaume Gelle, Georges Delaunay. Blind Separation of rotating machine signals using Penalized Mutual Information criterion and Minimal Distortion Principle. Mechanical Systems and Signal Processing, 2005, 19 (6), pp.1282 - 1292. 10.1016/j.ymssp.2005.08.028 . hal-01812922

\section{HAL Id: hal-01812922}

https://hal-enpc.archives-ouvertes.fr/hal-01812922

Submitted on 12 Jun 2018

HAL is a multi-disciplinary open access archive for the deposit and dissemination of scientific research documents, whether they are published or not. The documents may come from teaching and research institutions in France or abroad, or from public or private research centers.
L'archive ouverte pluridisciplinaire $\mathbf{H A L}$, est destinée au dépôt et à la diffusion de documents scientifiques de niveau recherche, publiés ou non, émanant des établissements d'enseignement et de recherche français ou étrangers, des laboratoires publics ou privés. 


\title{
Blind Separation of rotating machine signals using Penalized Mutual Information criterion and Minimal Distortion Principle
}

\author{
Mohammed EL Rhabi, Hassan Fenniri, \\ Guillaume Gelle and Georges Delaunay
}

CReSTIC-DéCom, University of Reims Champagne-Ardenne BP 103951687 Reims, Cedex 2, France

Preprint submitted to Mechanical Systems and Signal Processing Journal (2005)

\begin{abstract}
The Blind Separation problem of convolutive mixtures is addressed in this paper. We have developed a new algorithm based on a penalized mutual information criterion recently introduced in [El Rhabi et al., A penalized mutual information criterion for blind separation of convolutive mixtures, Signal Processing 84 (2004) 1979-1984] and which also allows to choose an optimal separator among an infinite number of valid separators that can extract the source signals in a certain sense according to the Minimal Distortion Principle. So, the minimisation of this criterion is easily done using a direct gradient approach without constraint on the displacements. Thus, our approach allows to restore directly the contribution of the sources to the sensor signals without post-processing as it is usually done. Finally, we illustrate the performances of our algorithm through simulations and on real rotating machine vibration signals.
\end{abstract}

Keywords: Fault detection; Condition monitoring; Signal Processing; Mechanical systems; Rotating machine; Blind source separation; Minimal Distortion Principle; Mutual Information

\section{Introduction}

In the last years, blind source separation (BSS) became a classical problem in signal processing due to the wide range of engineering applications that could benefit from such techniques. A general class of blind signal separation problem is the linear BSS where the mixing system is a linear time-dependent (or not) function. Such a model is named convolutive mixture and the correspondent separation, convolutive BSS. The principle of BSS is to transform a multivariate random signal into an ideal signal which has mutual independent components in the statistical sense (see [1,2]). So, BSS is achieved by maximising the distance between the probability density function (pdf) of the ideal signal and the pdf of the multivariate observed signal. Note that in practice, the pdfs are unknown and must be estimated. It has been shown in [3] that this distance can be easily related to the maximisation of a contrast function like the mutual information between the ideal signal and the observations. A new method is proposed in [4] to separate convolutive mixtures based on the minimisation of a delayed output mutual information where each mutual information term is minimised using 
the Marginal and the Joint score function. We have recently introduced in [5] a new algorithm based on this criterion plus a penalized term which achieves BSS regardless to the scale indeterminacy and also improves the robustness of the method. In this paper, we propose to modify the penalized algorithm [5] according to the Minimal Distortion Principle presented in [6]. So, this allows us to directly choose the optimal separator (in a sense define below) among an infinite number of valid separators that can extract the source signals. It is important to note that this optimal separator is uniquely determined and does not depend on the sources properties, it depends only on the mixing operator. Moreover, the proposed criterion allows us to use a direct gradient method without any constraint on the displacements and so a more efficient optimisation. Thus, we can restore directly the contribution of the sources to the sensor signals without using strong assumption on the mixing process [7] or a post-processing stage as it is usually done in numerous BSS applications (see [6] for example).

Subsequently, the proposed algorithm is applied to separate a mixture of vibration signals from rotating machines. Indeed, there exist a great interest in applying BSS in mechanical system for monitoring and diagnosis purpose because in the industrial case, the vibration measurements of one machine are, with respect to the superposition principle, always corrupted by their environment (other machines, noise...). In such application, the mixture clearly appears to be convolutive. Among the existing contribution considering BSS in the mechanical systems context, we can mention [7-13]. Gelle et al. [9] have suggested a blind approach for the reconstruction of primary sources and demonstrate that BSS method is a promising tool to pre-process the data in mechanical fault diagnosis applications.

Based on these results, we first illustrate the performances of our new algorithm through the experiment presented in [7] which deals with the two-dimensional BSS problem (2 sources/2 sensors). Next, we show that this new approach, contrary to the above mentioned is not restricted to the two-dimensional case. An example in the case of 3 sources $/ 3$ sensors is given to illustrate this.

This paper is organised as followed. Section 2 recalls the principle of BSS, and presents the model. Section 3 introduces the mutual information and the separation criterion. The new algorithm including the minimum distortion principle criterion is presented in Section 4. A discrete form of this criterion and its stochastic form are presented with some numerical results illustrating this work in Section 5. A conclusion of this work and some perspectives are given in the Section 6.

\section{Principle of convolutive BSS}

The mixing model can be introduced as follows (in the noise free case):

$$
\boldsymbol{x}(t)=\mathscr{A} * \boldsymbol{s}(t)
$$

where $*$ denotes the convolutive product, $\mathscr{A}$ is the mixing operator, $\boldsymbol{x}(t)$ the observation vector, and $\boldsymbol{s}(t)$ the independent component source vector. Then, the separating system is defined by

$$
\boldsymbol{y}(t)=\mathscr{B} * \boldsymbol{x}(t)
$$

where the vector $\boldsymbol{y}(t)$ is the output signal vector (estimated source vector) and $\mathscr{B}$ the separating operator. The system can be implemented as in Fig. 1

In the discrete form, Eqs. $(1,2)$ become:

$$
\begin{aligned}
& \boldsymbol{x}(n)=[\mathscr{A}(z)] \boldsymbol{s}(n)=\sum_{k} \mathscr{A}_{k} \boldsymbol{s}(n-k), \\
& \boldsymbol{y}(n)=[\mathscr{B}(z)] \boldsymbol{x}(n)=\sum_{k} \mathscr{B}_{k} \boldsymbol{x}(n-k),
\end{aligned}
$$

where $\mathscr{A}_{k}$ and $\mathscr{B}_{k}$ are, respectively, the corresponding $\mathscr{A}$ and $\mathscr{B} z$-transform matrices.

If we assume $\mathscr{A}$ is left-invertible and statistically independent sources, then the problem consists in finding $\mathscr{B}$ and $\boldsymbol{y}$ for a given $\boldsymbol{x}$ such that:

$$
\boldsymbol{y}(n)=[\mathscr{B}(z)] \boldsymbol{x}(n)=[\mathscr{B}(z)][\mathscr{A}(z)] \boldsymbol{s}(n),
$$

where $\mathscr{B}$ satisfies $[\mathscr{B}(z)][\mathscr{A}(z)]=[\mathscr{P} \mathscr{H}(z)]$, and $\mathscr{P}$ is a permutation operator and $\mathscr{H}$ a filtering operator. 


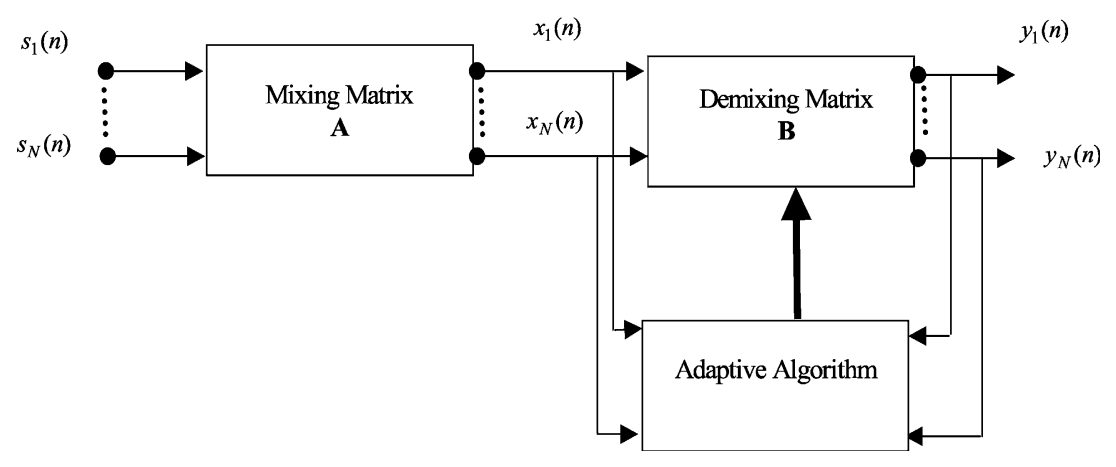

Fig. 1. Mixing and separating systems.

\section{Independence criterion}

Let $\boldsymbol{y}=\left(y_{1}, \ldots, y_{N}\right)^{\mathrm{T}}$ a random vector and consider $\boldsymbol{p}_{\boldsymbol{y}}$, the joint probability density function (joint pdf) and $p_{y i}, i \in\{1, \ldots, N\}$, the marginal probability density function of the $i$ th component of $\boldsymbol{y}$ (marginal pdf). In the BSS context, the mutual information can be written as follows:

$$
I(\boldsymbol{y})=\int_{\mathbb{R}^{N}} \boldsymbol{p}_{\boldsymbol{y}}(\boldsymbol{t}) \ln \left(\frac{\boldsymbol{p}_{\boldsymbol{y}}(\boldsymbol{t})}{\prod_{i=1}^{N} p_{y i}(\boldsymbol{t})}\right) \mathrm{d} \boldsymbol{t} .
$$

It is well known that Eq. (6) is non-negative and equal to zero if and only if the components are statistically independent.

With convolutive mixtures, it is easy to show that the independence between two scalar sources $y_{1}(n)$ and $y_{2}(n)$ (for all $n$ ) is not sufficient to separate the sources because we are dealing with random process and not random variables. That is why additional constraints must be stated to ensure the mutual independence of the output signal components $y_{1}(n), i \in\{1, \ldots, N\}$. To make it easier to understand, let us consider now a bidimensional random vector $\boldsymbol{y}(n)=\left(y_{1}(n), y_{2}(n)\right)$. The independence of the components $y_{1}(n)$ and $y_{2}\left(n^{\prime}\right)$ is needed for all $n$ and $n^{\prime}$ to ensure the separation, in a different way the independence of $y_{1}(n)$ and $y_{2}(n-m)$, for all $n$ and at all lags $m$.

Babaie-Zadeh et al. take this last remark into consideration and propose in [4] the minimisation of a separation criterion based on a tangential gradient. We proposed in [5] another approach which consists in overcoming the normalisation constraint by adding a penalization term to the criterion. This allows us to use a direct gradient method without any constraint on the displacements toward the optimum and so a more efficient optimisation. Unlike the Babaie-Zadeh et al. approach which uses implicitly a tangential gradient method, our algorithm is based on a global one, due to the addition of a penalization term. This normalisation term thus prevents the gradient from growing up and of course the algorithm from exploding (see [14] for more details on gradient methods).

However, it was emphasised previously that it is possible to restore the source original up to a linear filtering. It is possible, however in the case of instantaneous mixtures to reduce the shape indeterminacy in model (5) by setting a simple constraint on $\boldsymbol{x}$ where its components are generally assumed to have unit variance, naturally checked since the penalized term adds a signal normalisation feature to the algorithm.

Some other simple constraints could be assumed, for example on the diagonal terms of the mixing matrix $[\mathscr{A}(z)]$ which are usually supposed to be equal to unity. This assumption can be easily related to the fact that the sensors are as close to the sources as possible [7] which is suitable to fault detection and condition monitoring purposes.

Nevertheless, in the general case of more than two sources, this is not a sufficient condition and it is necessary to add other constraints to estimate the filtering indeterminacy effect of each source on each sensor. A very attractive approach is proposed in [6] Matsuoka et al. They introduced a principle, called the Minimal Distortion Principle which allows to choose an optimal separator among an infinite number of valid separators that can extract the source signals in a certain sense. The optimal choice is made such that the 
observed signals are the least subjected to distortion by the separator. Namely, a valid separator [ $\mathscr{B}(z)]$ is defined as follows:

$$
[\mathscr{B}(z)]=[\mathscr{P}(z)][\mathscr{D}(z)],
$$

where $[\mathscr{P}(z)]$ is a permutation matrix, $[\mathscr{D}(z)]$ is an arbitrary non-singular diagonal matrix.

So, we can state the Minimal Distortion Principle as follows: the separator must be chosen such as its outputs become as close to the sensors outputs as possible.

In other words, the optimal separator $\left[\mathscr{B}(z)^{\mathrm{opt}}\right]$ is the valid separator which minimizes:

$$
E\left[\|\boldsymbol{y}(n)-\boldsymbol{x}(n)\|^{2}\right]=E\left[\|[\mathscr{B}(z)] \boldsymbol{x}(n)-\boldsymbol{x}(n)\|^{2}\right],
$$

where $\|\cdot\|$ represents the Euclidean norm.

So, according to the Minimum Distortion Principle, and from the Penalized Mutual Information criterion recently introduced in [5], we propose as independence criterion:

$$
\tilde{J}_{\mathrm{MD}}(n)=\sum_{\boldsymbol{q}} I\left(\boldsymbol{y}^{\boldsymbol{q}}(n)\right)+\lambda \sum_{i=1}^{N} \sum_{\boldsymbol{q}}\left(E\left[\left(\boldsymbol{y}_{i}^{\boldsymbol{q}}(n)-E\left[\boldsymbol{y}_{\boldsymbol{i}}^{\boldsymbol{q}}(n)\right]\right)^{2}\right]-1\right)^{2}+\gamma \sum_{\boldsymbol{q}} E\left[\left\|\boldsymbol{y}^{\boldsymbol{q}}(n)-\boldsymbol{x}^{\boldsymbol{q}}(n)\right\|^{2}\right],
$$

where $\boldsymbol{x}^{q}(n)=\left(x_{1}\left(n-q_{1}\right), x_{2}\left(n-q_{2}\right), \ldots, x_{N}\left(n-q_{N}\right)\right)^{\mathrm{T}}$ when the vector $\boldsymbol{x}(n)$ is the random observed vector and $\boldsymbol{q}$ is a vector of integers. It is trivial to show that criterion (8) reaches its minimum with normalised independent component outputs which are close to the observation outputs, since we choose $\lambda>0$ and $\gamma>0$. Indeed, the Minimal Distortion Principle forces the estimated sources to be as close as possible to its contribution on sensors, but the normalisation process is not necessary ensured; nevertheless, this normalisation is very important to avoid the algorithm from numerically exploding. To overcome this problem, we retain from [5] the normalisation term in criterion (8).

\section{The algorithm}

In this section, we apply the gradient approach to separate convolutive mixtures based on the minimisation of criterion (8). To separate the sources by means of FIR filters with maximum degree $p$, the de-mixing system will be:

$$
\boldsymbol{y}(n)=\sum_{k=0}^{p} \mathscr{B}_{k} \boldsymbol{x}(n-k),
$$

where the infinite summation in Eq. (4) is replaced by a finite one.

To approximate the matrices $\mathscr{B}_{k}$ leading to estimated source outputs, we calculate the gradients of $\tilde{J}$ with respect to each $\mathscr{B}_{k}$.

So, the derivation leads to multivariate score functions, namely the Joint Score Function (JSF), the Marginal Score Function (MSF) and the Score Function Difference (SFD) defined, respectively, by

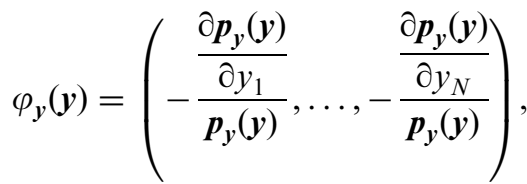

$$
\begin{aligned}
& \psi_{\boldsymbol{y}}(\boldsymbol{y})=\left(-\frac{\boldsymbol{p}_{y_{1}}^{\prime}\left(y_{1}\right)}{\boldsymbol{p}_{y_{1}}\left(y_{1}\right)}, \ldots,-\frac{\boldsymbol{p}_{\boldsymbol{y}_{N}}^{\prime}\left(y_{N}\right)}{\boldsymbol{p}_{\boldsymbol{y}_{N}}\left(y_{N}\right)}\right),
\end{aligned}
$$

and $\beta_{y}(\boldsymbol{y})=\psi_{y}(\boldsymbol{y})-\varphi_{y}(\boldsymbol{y})$.

\subsection{The gradient}

Let $\mathscr{B}_{k}$ a matrix, $\mathscr{E}$ a "small" matrix, to calculate the gradient with respect to $\mathscr{B}_{k}$ of $\tilde{J}$. We set $\hat{\boldsymbol{B}}_{k}=\mathscr{B}_{k}+\mathscr{E}$ a matrix in a neighborhood of $\mathscr{B}_{k}$. 
From Eq. (9), we have by definition

$$
\hat{\boldsymbol{y}}(n)=[\hat{\boldsymbol{B}}(z)] \boldsymbol{x}(n)=\boldsymbol{y}(n)+\mathscr{E} \boldsymbol{x}(n-k) .
$$

Setting $\boldsymbol{h}(n)=\mathscr{E} \boldsymbol{x}(n-k)$, we have

$$
\hat{\boldsymbol{y}}^{\boldsymbol{q}}(n)=\boldsymbol{y}^{\boldsymbol{q}}(n)+\boldsymbol{h}^{\boldsymbol{q}}(n) \text {. }
$$

Then if we consider $\tilde{J}_{\mathrm{MD}}$ defined by Eq. (8), we can state the following result:

$$
\begin{aligned}
\tilde{J}_{\mathrm{MD}}\left(\hat{\boldsymbol{y}}^{q}(n)\right)-\tilde{J}_{\mathrm{MD}}\left(\boldsymbol{y}^{q}(n)\right)= & \left\langle\mathscr{E}, E\left\{\beta_{\boldsymbol{y}^{q}}^{-q}(n) \boldsymbol{x}(n-k)^{\mathrm{T}}\right\}\right\rangle+\lambda\left\langle\mathscr{E}, E\left\{\boldsymbol{w}(n) \boldsymbol{x}(n-k)^{\mathrm{T}}\right\}\right. \\
& \left.+2 \gamma E\left\{(\boldsymbol{y}(n)-\boldsymbol{x}(n)) \boldsymbol{x}(n-k)^{\mathrm{T}}\right\}\right\rangle+\mathrm{o}(\mathscr{E}),
\end{aligned}
$$

where $\boldsymbol{w}=\left(w_{1}, \ldots, w_{N}\right)$ with $\boldsymbol{w}_{i}=4\left(E\left[\boldsymbol{y}_{i}^{q 2}\right]-1\right) y_{i} \mathrm{o}(\mathscr{E})$ denotes higher-order terms in $\mathscr{E}$ and $\langle C, D\rangle=$ trace $\left(C D^{\mathrm{T}}\right)$ is the matrix inner product (associated with the Schur norm).

It follows from the previous result:

$$
\frac{\partial \tilde{J}_{\mathrm{MD}}\left(\boldsymbol{y}^{q}(n)\right)}{\partial \hat{\boldsymbol{B}}_{k}}=E\left\{\beta_{\boldsymbol{y}^{q}}^{-\boldsymbol{q}}(n) \boldsymbol{x}(n-k)^{\mathrm{T}}+\lambda \boldsymbol{w}(n) \boldsymbol{x}(n-k)^{\mathrm{T}}\right\}+2 \gamma E\left\{(\boldsymbol{y}(n)-\boldsymbol{x}(n)) \boldsymbol{x}(n-k)^{\mathrm{T}}\right\} .
$$

\subsection{Algorithm}

From Eq. (11), we derived the following algorithm:

for $k=0, \ldots, p$ and given $\hat{\boldsymbol{B}}_{k}^{0}$ do

$$
\hat{\boldsymbol{B}}_{k}^{n}=\hat{\boldsymbol{B}}_{k}^{n-1}-\mu \frac{\partial \tilde{J}_{\mathrm{MD}}}{\partial \hat{\boldsymbol{B}}_{k}^{n-1}},
$$

update $\boldsymbol{y}^{n}$ such as:

$$
\boldsymbol{y}^{n}=\left[\hat{\boldsymbol{B}}^{n}(z)\right] \boldsymbol{x}(n)=\sum_{k=0}^{p} \hat{\boldsymbol{B}}_{k}^{n} \boldsymbol{x}(n-k),
$$

return $y^{n}$

There are some practical problems associated with the implementation of this algorithm. It requires the estimation of the score functions (10) which are easily approximated by a polynomial or Pham's approach (see [15]). However, we can notice that the numerical cost of the algorithm depends strongly on this approximation. We can also notice that from a theoretical viewpoint, it is possible to handle convolutive mixtures with long impulse responses but the algorithm becomes very expensive and time consuming.

\section{Numerical results}

Three examples are presented to illustrate the performances of our algorithm. The separation criterion (8) is used in its discrete form, i.e. the finite summation over $\boldsymbol{q}_{i} \in\{-M, \ldots, M\}$ takes the place of the infinite one over $\boldsymbol{q}_{i} \in \mathbb{Z}$, where $M=2 p$ ( $p$ is the maximum degree of the separating filters). Since this criterion is computationally expensive, we use its stochastic version. In other words, at each iteration, $m$ is randomly chosen from the set $\{-M, \ldots, M\}$.

\subsection{Example 1. Blind Separation of convolutive mixtures of simulated signals}

In order to illustrate the performance of criterion (8), we dealt with 3 observations obtained by a convolutive mixture of three real, non-gaussian and independent sources with zero means. The separation 
performance is given using the output Signal-to-Noise Ratio (SNR) defined by

$$
S N R_{i}=10 \log _{10}\left(\frac{E\left[y_{i}^{2}\right]}{E\left[\left(\left.y_{i}\right|_{\left\{s_{i}=0\right\}}\right)^{2}\right]}\right),
$$

where $\left.y_{i}\right|_{\left\{s_{i}=0\right\}}=\left\{([\mathscr{B}(z)][\mathscr{A}(z)] s(n))_{i}\right\}_{s_{i}=0}$.

The mixing operator $[\mathscr{A}(z)]$ is randomly chosen. The length of the observation signals is equal to 500 samples, and the SFD are estimated using the polynomial estimator. The experiment is repeated 20 times with different random sources realisations.

Fig. 2 shows the averaged SNRs in $\mathrm{dB}$ versus iterations for the penalized algorithm coupled with the Minimal Distortion Principle.

In the simulation, the adapting step is equal to $\mu=0.1$, the penalization parameters are equal to $\lambda=1 e-4$ and $\gamma=0.01$. We clearly notice that the convergence of this algorithm is quite stable, and that it provides a good quality of separation (up to $20 \mathrm{~dB}$ ). Note also that this algorithm is able to separate mixtures independently of the sources number.

\subsection{Example 2. Blind Separation of convolutive mixtures of experimental signals}

The purpose of this part is to show the performance of our algorithm in accordance with the experimental test proposed by Gelle et al. [7]. We briefly recall the experimental context which was realised on a test bench carrying two dc motors $(1.4$ and $1.1 \mathrm{~kW})$ with different rotation speeds. The two motors were fixed on the same structure as in Fig. 3. Two accelerometers were glued on each motor to measure vibrations. The problem illustrated by this experiment is one of a factory in which two rotating machines operate simultaneously, but each machine must be diagnosed separately. Thus according to the superposition principle, signals from the other machine disrupt signals received by sensors placed on each machine. There is a great interest in the use of BSS methods as part of the diagnostic process because BSS should free us from noisy environment; that is restoring on each sensor the signature of its own machine without having to stop the machines which would be damaging to the production. For this purpose, BSS can be viewed as a pre-processing step (de-noising) that improves the diagnosis. Traditional methods of fault detection could then be applied to the specific signatures of the system to be diagnosed. When treating real recording, it is very difficult to measure the separation quality. Here, prior knowledge about the sources was used; that is, harmonic frequencies in relation to the

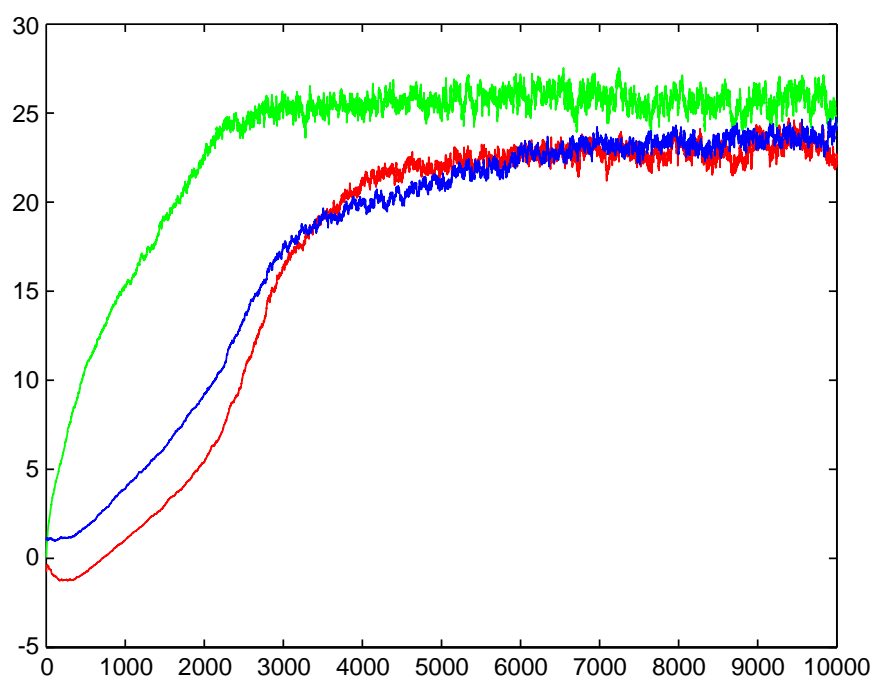

Fig. 2. Averaged output SNRs versus iterations with polynomial's estimation of the SFD. We use 3 random iid sources and a mixing system of length 12 (i.e. each mixing filter is a twelve-order FIR filter). 


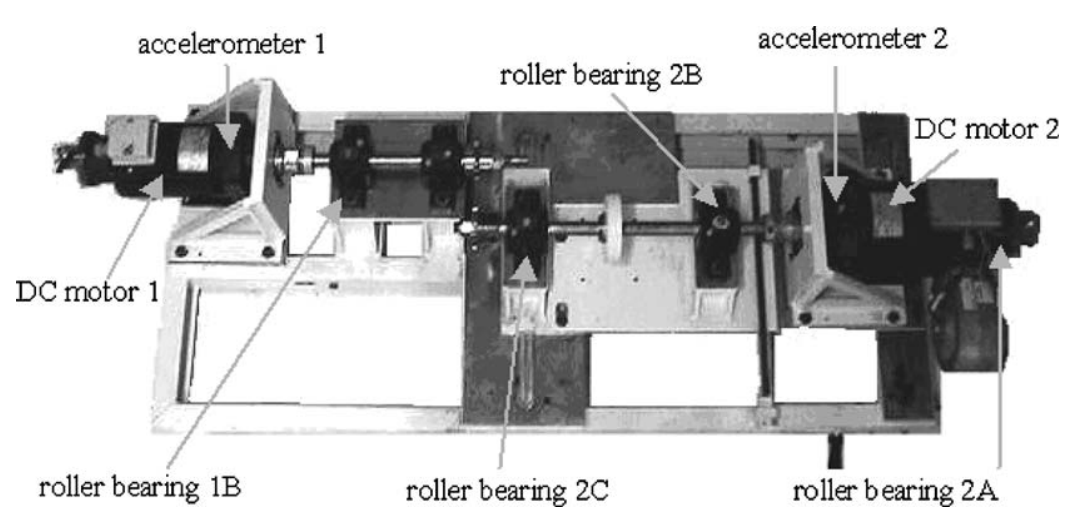

Fig. 3. Test bench.
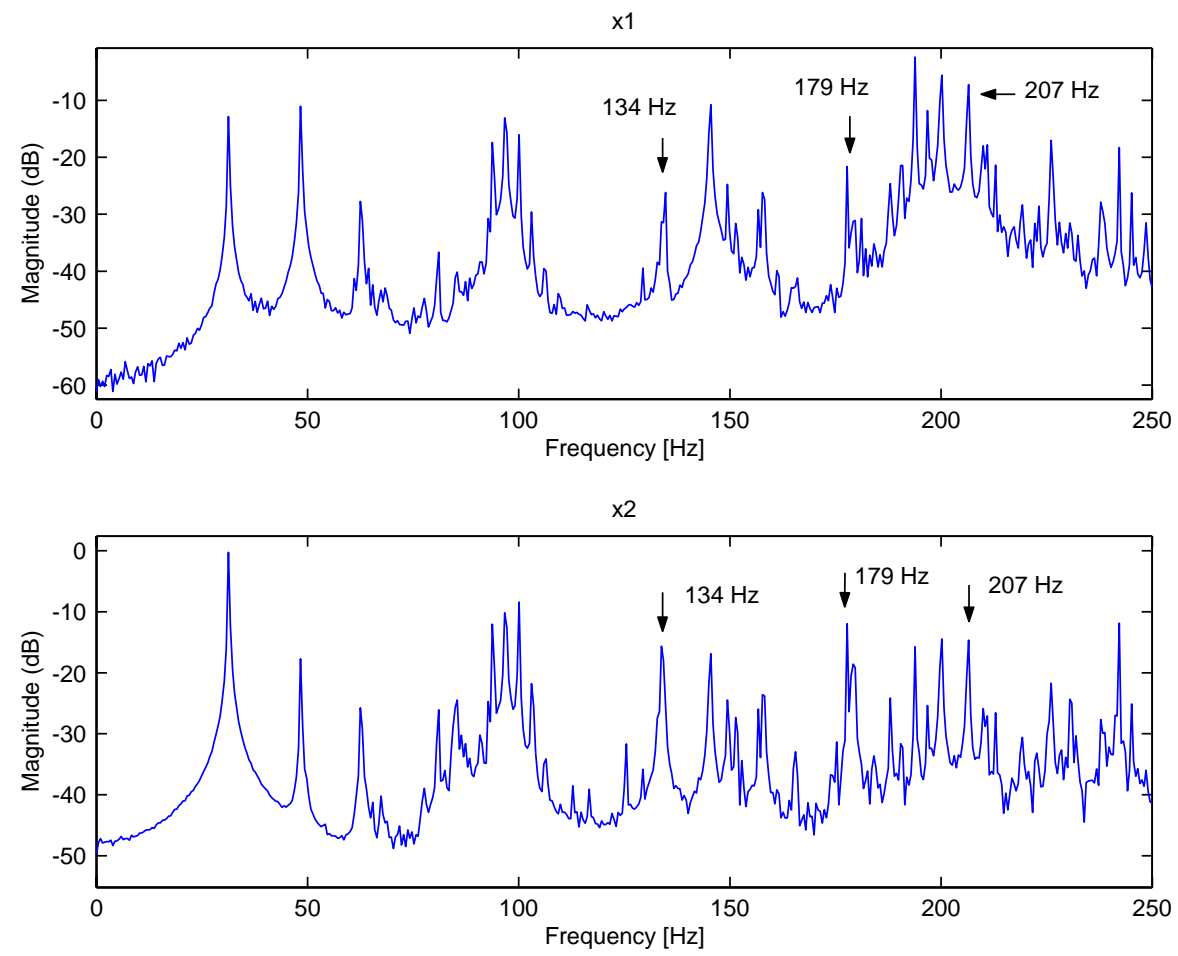

Fig. 4. PSD of the recorded signals.

mechanical components as well as the signals recorded on each source separately in the real environment (the reference). The rotation speed of the two motors are set to $48.5 \mathrm{~Hz}$ for motor $1(1.1 \mathrm{~kW})$ and $31.5 \mathrm{~Hz}$ for motor $2(1.4 \mathrm{~kW})$. Motor 1 is fed by signal-phase wiring (rectified) which provides $100 \mathrm{~Hz}$ for the fundamental frequency plus the harmonics. The motor 2 is fed by three-phase wiring (rectified) which presents 100, 200 and $300 \mathrm{~Hz}$ frequencies.

Each motor is fitted out with two single-row bearings (6203 RS C3) and drives a main shaft filled with two self-aligning roller bearings (2207 KTV C3). Roller bearings 2A, 2C and IB were found to be faulty and to induce four defect frequencies at $134 \mathrm{~Hz}$ (outer race fault on 2C), $179 \mathrm{~Hz}$ (outer race fault on $2 \mathrm{~A}$ ), $207 \mathrm{~Hz}$ (outer race fault on $1 \mathrm{~B}$ ) and $210 \mathrm{~Hz}$ (inner race fault on $2 \mathrm{~B}$ ).

Fig. 4 presents the Power Spectral Density (PSD) estimated using Welch averaged method of the two records measured on each sensor. To illustrate the potential of BSS in bearing fault detection, we stress in this 
example one clearly identified fault related to the $207 \mathrm{~Hz}$ frequency. This fault is positioned on the outer race of the axe driving the roller bearing of the motor 1 (bearing 1B).

Fig. 5 clearly shows that not only the rotating frequencies plus harmonics (position and magnitude) are restored, respectively, to each source but also that as in [9], this fault-related frequency $(207 \mathrm{~Hz})$ is associated to the right source.

However, the introduction of the Minimal Distortion Principle relatively to the approach in [9] allows to obtain better results on two other faults connected with the bearing $2 \mathrm{~B}(210 \mathrm{~Hz})$ and with the bearing $2 \mathrm{~A}$ $(179 \mathrm{~Hz})$. Moreover, we also notice than the fifth harmonic of the rotation speed of motor $1(242.5 \mathrm{~Hz})$ is enhanced with the MDP approach. We can also point out that contrary to some previous results presented in [16] no frequency channels are permuted. So, BSS can be viewed as an efficient pre-processing step, which makes easier and enhances the detection and the monitoring of the mechanical system to be diagnosed.

\subsection{Example 3. Blind Separation of artificial mixture of three real rotating machines vibration signals}

We consider in this section the separation of 3 sources. As we do not have a real experiment with more than two sources, we simulate 3 observations signals by an artificial convolutive mixtures of 3 real rotating machines vibrations signals $s_{1}, s_{2}, s_{3}$ (see Fig. 6). The source signal $s_{1}$ is measured by an accelerometer fixed on motor 1 (when motor 2 is stopped) and $s_{2}$ is measured by an accelerometer fixed on motor 2 (when motor 1 is stopped), the rotation speed are, respectively, set to 22 and $38 \mathrm{~Hz}$. The third source signal $s_{3}$ is measured by an accelerometer fixed on motor 2 where the rotation speed is taken to $31.25 \mathrm{~Hz}$.

The mixing system $\mathscr{A}$ is randomly chosen. Each mixing filter is FIR of length 12 . The PSD of the mixtures can be shown on the Fig. 7.

The results shown in Fig. 8 indicate that this approach gives satisfactory results for the 3 motors rotating frequencies plus harmonics. Moreover, as in the previous example we can point out that no frequency channel
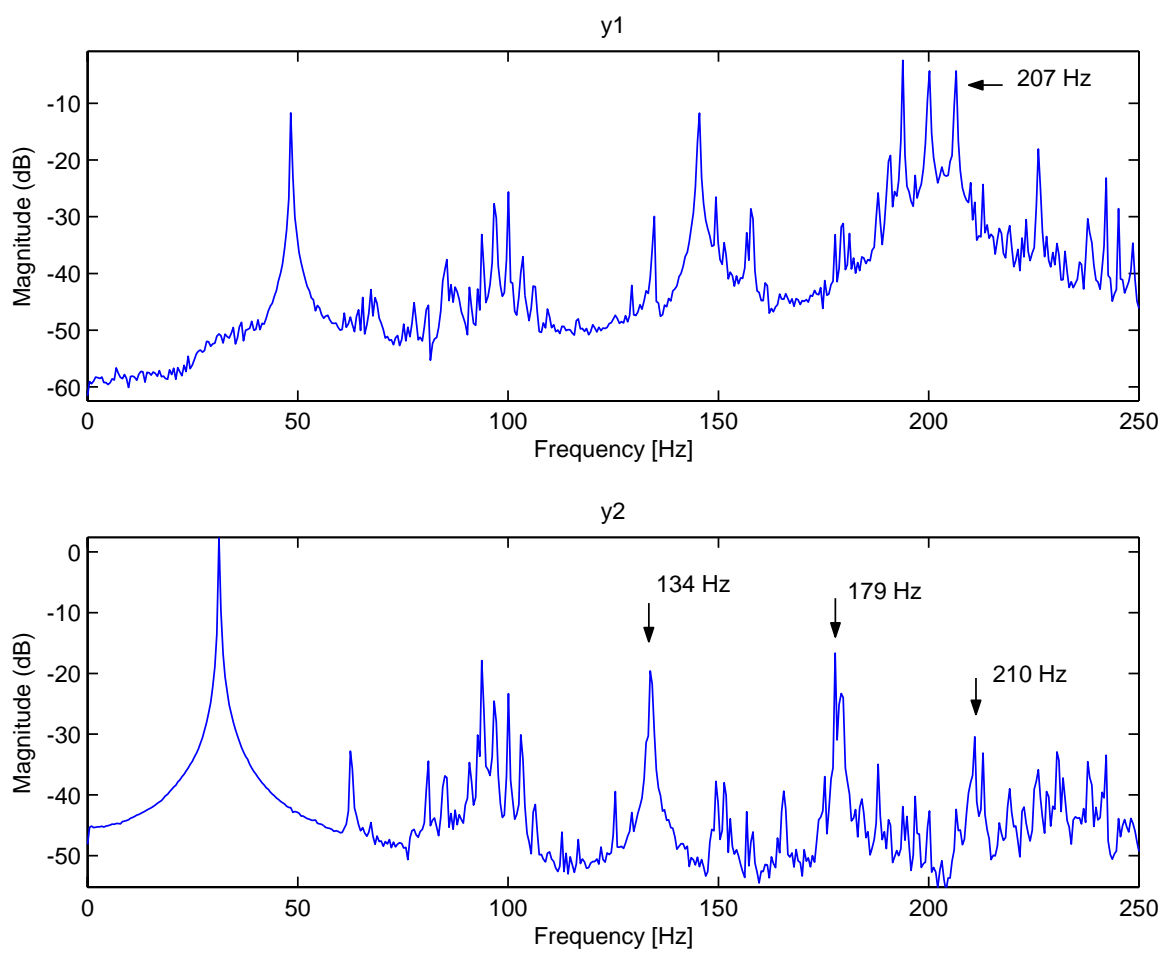

Fig. 5. PSD of the estimated sources. The score functions are estimated using a polynomial estimation, the maximum degree of the FIR taken equals to $50(\mathrm{p}=50, \mathrm{M}=100)$. The adapting step-size, and the penalized parameters are, respectively, taken to $\mu=0.1, \lambda=0.0001$ and $\gamma=0.01$. 

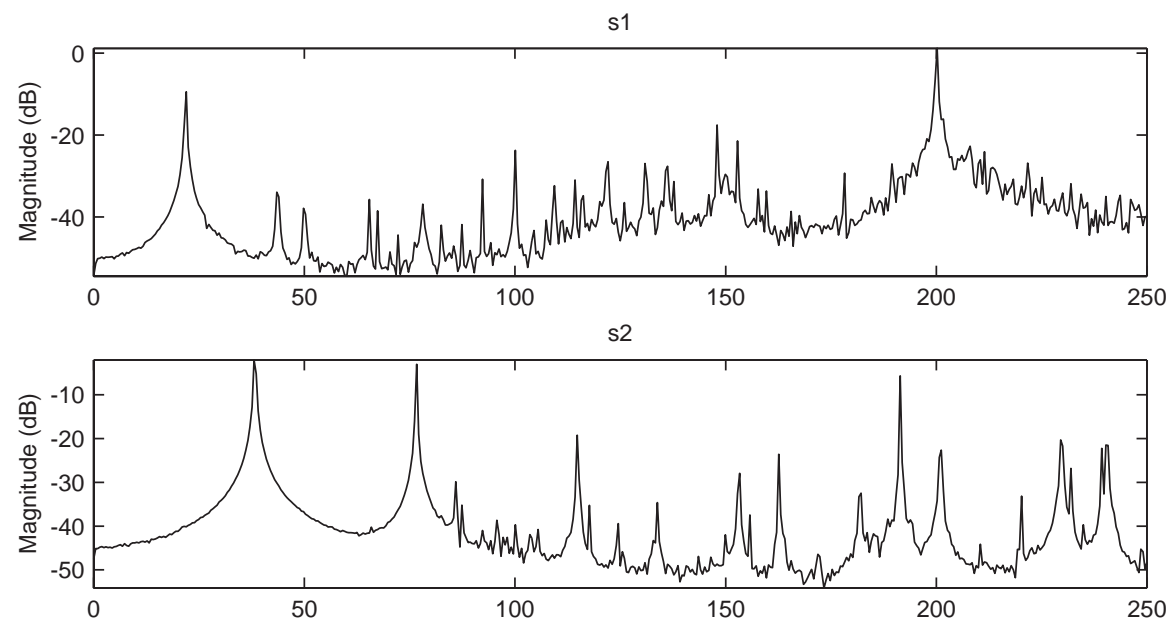

s3

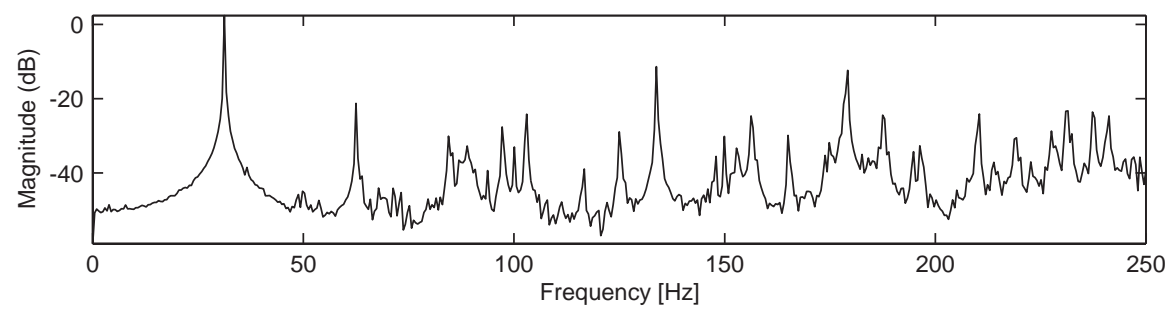

Fig. 6. PSD of the three real rotating machines (sources) $s_{1}, s_{2}, s_{3}$.
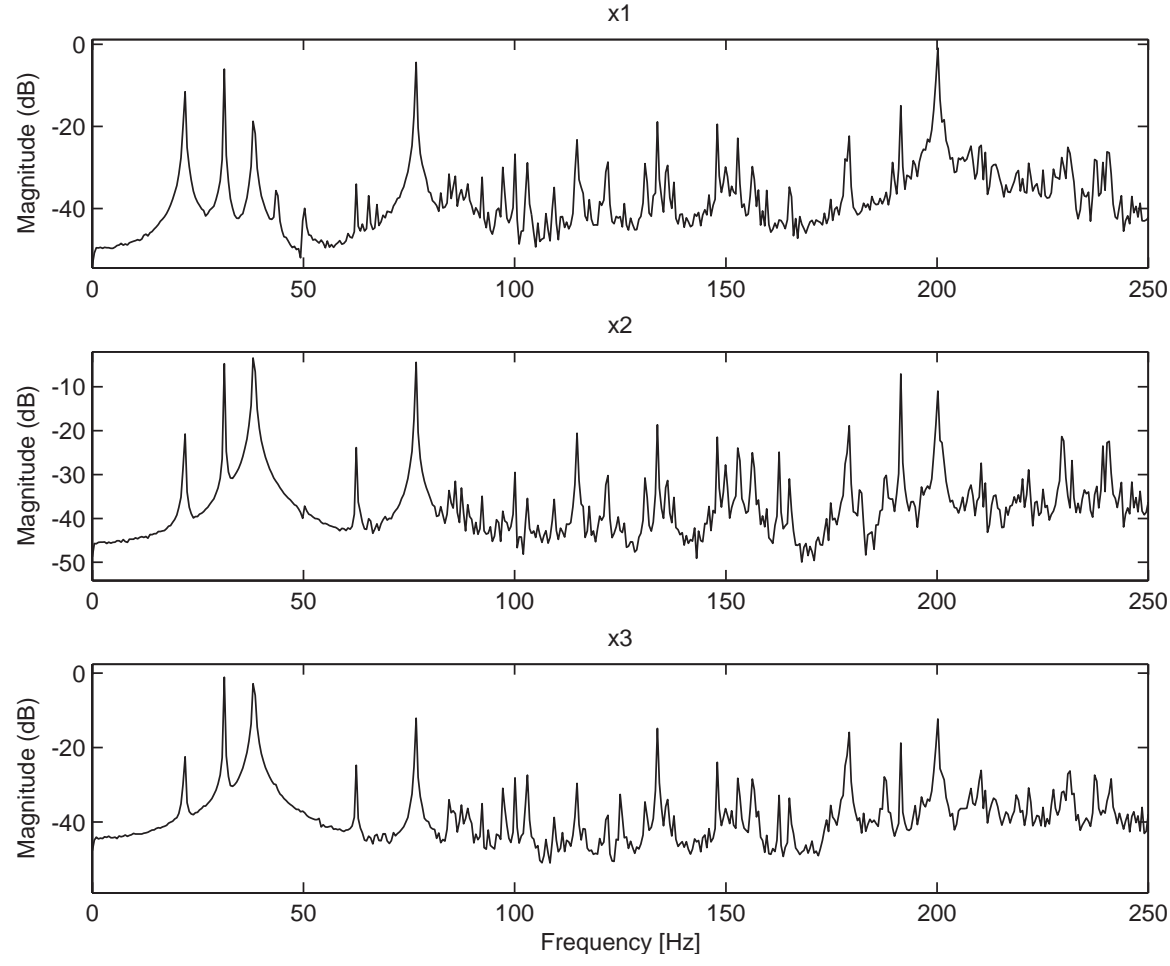

Fig. 7. PSD of the three observed signals. 

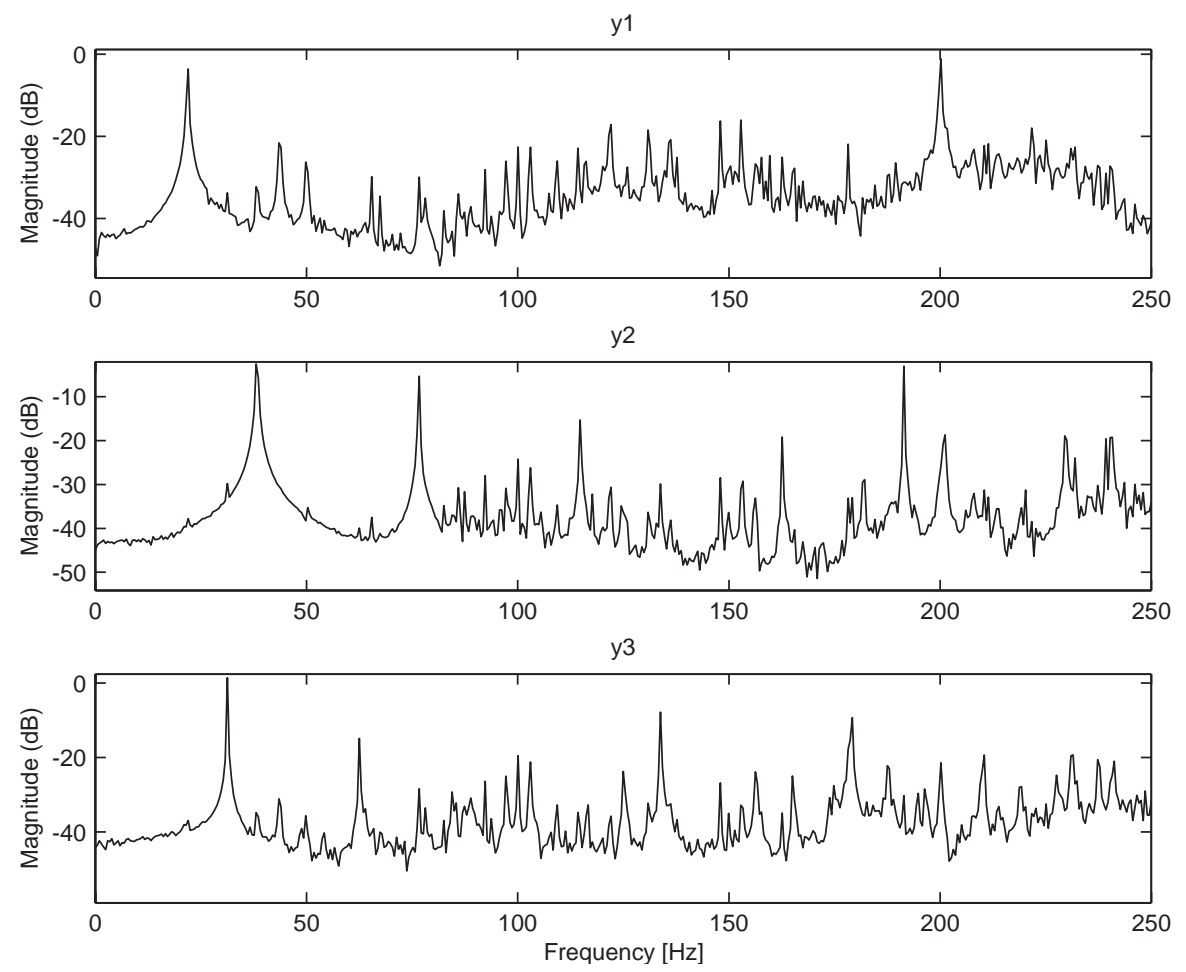

Fig. 8. PSD of the estimated sources. The score functions are estimated using a polynomial estimation, the maximum degree of the FIR taken equals to $12(p=12, M=24)$. The adapting step-size, and the penalized parameters are, respectively, taken to $\mu=0.1, \lambda=0.0001$ and $\gamma=0.01$.

is permuted. We can also notice that the output SNRs for this example is equal to $25 \mathrm{~dB}$ for each estimated source which shows the robustness of the present algorithm (see Fig. 2).

\section{Conclusion}

A new convolutive BSS algorithm based on the minimisation of a mutual information criterion penalized by an additional Minimal Distortion Principle term is presented. This algorithm is implemented by a global gradient method (without any constraint on its displacement) which ensures an efficient optimisation.

The introduction of the Minimal Distortion Principle allows the mitigation of the filtering indeterminacy inherent to the BSS problem, the optimal separator is only "optimal" in the sense that the estimated sources are the least subjected to distortion among the set of all the valid separators. We show that the implementation of such approach improves appreciably the quality of the sources separation. The simulation results presented in Section 5.3 have shown that this approach is proving to be efficient in terms of stability and SNR's performances. Future work will be oriented towards a real mechanical systems signal processing with many sources.

\section{References}

[1] P. Comon, Independent component analysis, a new concept?, Signal Processing 36 (3) (1994) 287-314.

[2] A. Hyvarinen, J. Karhunen, E. Oja, Independent Component Analysis, Wiley, New York, 2001.

[3] Jean-François Cardoso (Ed.), Blind signal separation: statistical principles, Proceedings of the IEEE 90 (8) (1998) 2009-2026, special issue on blind identification and estimation, R.-W. Liu and L. Tong (Eds.). 
[4] M. Babaie-Zadeh, C. Jutten, K. Nayebi, Separating convolutive mixtures by mutual information minimization, in: Proceedings of IWANN2001, Granada, Spain, June 2001, pp. 834-842.

[5] M. El Rhabi, G. Gelle, H. Fenniri, G. Delaunay, A penalized mutual information criterion for blind separation of convolutive mixtures, Signal Processing 84 (2004) 1979-1984.

[6] K. Matsuoka, Y. Ohba, Y. Toyota, S. Nakashima, Blind separation for convolutive mixture of many voices, International Workshop on Acoustic Echo and Noise Control (IWAENC2003), September 2003, Kyoto, Japan.

[7] G. Gelle, M. Colas, C. Servière, Blind source separation: a new pre-processing tool for rotating machines monitoring?, IEEE Transactions on Instrumentation and Measurement 52 (2003) 790-795.

[8] H. Fenniri, M. El Rhabi, G. Gelle, G. Delaunay, A separating convolutive mixtures by a penalized mutual information contrast. Application to a bearing fault detection, Fifth International Conference Acoustical and Vibratory Surveillance Methods and Diagnostic Techniques, 11-13 October 2004, Cetim, Senlis Fr.

[9] G. Gelle, M. Colas, G. Delaunay, Blind source separation applied to rotating machines monitoring by acoustical and vibration analysis, Mechanical Systems and Signal Processing 14 (3) (2000) 427-442.

[10] A. Ypma, A. Leshem, R.P.W. Duin, Blind separation for rotating machine sources: bilinear forms and convolutives mixtures, Neurocomputing 49 (2002) 349-368.

[11] A. Ypma, A. Leshem, R.P.W. Duin, Blind separation of rotating machine sources: bilinear forms and convolutive mixtures, Neurocomputing 49 (1-4) (January 2003) 349-368 (special issue on ICA/BSS).

[12] Adriana Dapena, Christine Servière, A simplified frequency domain approach for blind separation of convolutive mixtures, in: Proceedings of ICA, San Diego, USA, 2001, pp. 569-574.

[13] J. Antoni, F. Guillet, M. El Badaoui, F. Bonnardot, Blind separation of convolved cyclostationary processes, Signal Processing 85 (2005) 51-66.

[14] P.G. Ciarlet, Introduction à l'Analyse Numérique Matricielle et l'Optimisation, Cambridge University Press, Cambridge (Masson, Paris, 1982, English translation: 1989).

[15] D.T. Pham, Fast algorithm for estimating mutual information, entropies and score functions, in: Proceeding of ICA 2003 Conference. April 2003, Nara, Japan.

[16] G. Gelle, M. Colas, H. Fenniri, G. Delaunay, Blind source separation applied to mechanical system monitoring and fault detection: some results and comments, Fourth International Conference Acoustical and Vibratory Surveillance Methods and Diagnostic Techniques, Compiegne, France, 2001. 